Review Article

\title{
Evidence-Based Guideline on Critical Patient Transport and Handover to ICU
}

\author{
Tesfaye Belaneh Agizew, Henos Enyew Ashagrie iD, Habtamu Getinet Kassahun, \\ and Mamaru Mollalign Temesgen
}

\author{
Department of Anesthesia, College of Medicine and Health Sciences, University of Gondar, Gondar, Ethiopia \\ Correspondence should be addressed to Henos Enyew Ashagrie; henos.enyew2007@gmail.com
}

Received 2 November 2020; Revised 8 April 2021; Accepted 16 April 2021; Published 6 May 2021

Academic Editor: Ronald G. Pearl

Copyright (C) 2021 Tesfaye Belaneh Agizew et al. This is an open access article distributed under the Creative Commons Attribution License, which permits unrestricted use, distribution, and reproduction in any medium, provided the original work is properly cited.

\begin{abstract}
The perioperative period is a time in which significant physiological change occurs. Improper transfer of information at this point can lead to medical errors. Planning and preparation for critical patient transport to ICU is vital to prevent adverse events. Critical patient transport to ICU must be as safe as possible and should not cause additional risks. It needs good communication, planning, and appropriate staffing with standard monitoring. Evidence shows inconsistency and variability on the use of standardized protocols during critical patient transfer and handover to the ICU. There is a variety of controversial approaches about the need of sedation, use of end-tidal $\mathrm{CO}_{2}$ monitoring, and manual versus mechanical ventilation based on different evidence. The objective of this review was to recommend safer options of critical patient transfer to the ICU that help reduce patient morbidity and mortality. Methods. Google Scholars, PubMed through HINARI, and other search engines were used to search high-quality evidence that help reach appropriate conclusions. Discussion. Critical patient transfer and handover to ICU is a complex procedure that needs experienced hands, availability of appropriate team members, standard monitoring, and necessary emergency and patient-specific medications. Appropriate and adequate transfer of patient information to the receiving team decreases patient morbidity and mortality when the transfer team uses standardized checklist. Conclusion. Involvement of senior physicians, use of standard monitoring, and appropriate transfer of information have been shown to decrease critical patient morbidity and mortality.
\end{abstract}

\section{Introduction}

The postoperative period is a time in which significant physiological change occurs, and this is the time in which the patient recovers from the acute instabilities resulting from anesthesia and surgery. Inadequate and improper transfer of information at this point can lead to medical errors [1]. Transporting patients is a risky procedure, and it requires good communication, planning, and appropriate staffing. Any patient who requires transportation must be effectively stabilized before departure. Planning and preparation are vital to prevent the adverse event [2]. Common postoperative physiologic disorders include nausea and vomiting, oliguria, hypoxia, hypotension, hypothermia, bleeding delirium, pain, and delayed awakening [3].
The main purpose of transporting critically ill patients to the intensive care unit (ICU) is to improve patient prognosis or to reduce morbidity and mortality. Increasing monitoring capabilities are vital during critical patient transportation, and the transport must be as safe as possible and should not cause additional risks [4]. Monitoring battery supply problem, lack of same emergency drug, unplanned intubation, accidental extubation, oxygen supply failure, transport ventilator malfunction, poor transport team, and accidental intravenous line dislodgment may occur on transporting critical patients [5].

One study in France showed that $45.5 \%$ of critical patients suffered one or more adverse events during transport to ICU. Risk factors for complication were ventilation with positive end-expiratory pressure $>6 \mathrm{~cm} \mathrm{H} 2 \mathrm{O}$, sedation before transport, and fluid loading [6]. The incidence of arterial 
desaturation is highest during this period so that functional pulse oxymetery is mandatory during this time [7].

Patient handover is the transmission of information, professional responsibility, and accountability between individuals and teams [8]. Some study shows that anesthesiologists and ICU nurses had different beliefs concerning the content of information and opinions on what information needs to be reported [9]. The handover and transport of critically ill pediatric patients require communication amongst multiple disciplines. Poor communication is associated with nearly $64 \%$ to $70 \%$ of sentinel events in the hospital (The Joint Commission, 2015) [10]. Transport and handovers between operating theatres and intensive care departments pose unique challenges [11]. Transfer of critical patients from the operation theatre to ICU is considered to be a high-risk period for the development of vital sign derangement [12].

Guidelines and minimum standards should be available in performing a uniform practice. We commonly encounter controversy on which information, for whom, at what time should be transferred [13].

Currently, some research studies show that large numbers of critically ill patients who encountered significant risks were poorly managed during transport and handover [14]. One study conducted in USA identified that postoperative handover is informal, unstructured, and inconsistent with often incomplete transfer of essential information [15]. Patient transfer and handover to ICU is extremely complex and is often characterized by incomplete, unstructured, and unsafe practices [16]. Operation theatre to ICU hand offs are known sources of medical error because of poor handover practice [17]. Henceforth, we need to review evidence that focuses on current practices and to recommend safer options of critical patient transfer to the ICU that help reduce patient morbidity and mortality.

\section{Methodology}

A comprehensive search for evidence was conducted at Pub Med/PMC, Google Scholar, and Cochrane database review using appropriate filtering method. Key terms like "critical patient," "intensive care unit," "transfer," "handover," and "transportation" were used in various combinations.

After collecting reasonable amount of evidence (Table 1), appraisal of quality of studies by using different institutional appraisal checklists was used to categorize evidence into levels. Final conclusions and recommendations were reached based on the WHO 2011 level of evidence (Table 2). Using PRISMA 2009 flow diagram, searching of systematic reviews, RCTs, evidence-based guidelines, and cohort studies were filtered for the development of guideline (Figure 1).

\section{Results}

One systematic review showed patient transfer and handover was characterized by poor teamwork and communication. The review reported that patients arrive in a compromised state, and there were technical errors, unstructured processes, unclear procedures, interruptions, and distractions during handover. It recommended that all team members should be available, complete urgent task before transfer of information, and allow patient-specific handover and all things must be cleared out [8].

Another systematic review described postoperative handovers as a complex work process challenged by interruptions, time pressure, PACU staff turnover, and a lack of supporting framework. Interventional studies introduced standardized handover tools in combination with environmental changes, resulting in better flow of information, and the main purpose of handover is to create a cognitive picture of the patient that leads to effective decision-making [18].

One systematic review recommended the stabilization, maintaining secured airway, using minimum monitoring, inserting intravenous line, and sedating and paralyzing of intubated patients before transferring. The review added that the patient should be mechanically ventilated, and the team should have in hand of emergency drugs, securing the patient within the transport trolley, and finally filling and signing of the transport form by the consultant [8]. A study conducted at University of Oxford classifies patient handover to ICU into four phases: prehandover, equipment and monitoring handover, information handover, and discussion and plan handover [19]. Studies conducted in France found deficits on information transfer regarding type of anesthesia, type of procedure, duration of surgery, medication history, any intraoperative event, fluid input/output, and postoperative special concern [1].

One systematic review suggests that checklist-centered interventions may be effective in improving the quality and completeness of critical patient handover. Hardwiring within the hospital system through use of uniform tools and methods allow chances to ask questions, reinforce quality and measurement through integration into medical governance and ongoing audit, as well as education and training in the conduct of successful handovers [20].

Patients with lower probability of recovery from anesthesia, those with respiratory failure and need invasive ventilation, patients who require life support for organ failure [21], and patients in of need of intensive monitoring, continuous renal replacement therapies, and invasive hemodynamic monitoring are considered critically ill individuals [22].

Common steps during patient transport includes decision to transport, planning to transport, choice of appropriate transport team, choice of appropriate mode of transport, selection of appropriate monitoring and equipment, prediction of possible complication and management options, and implementing the transport [23]. Guidelines showed that the minimum standards of monitoring required during critical patient transport include appropriate trained and skilled staff, continuous cardiac rhythm (ECG) monitoring, noninvasive blood pressure, oxygen saturation $\left(\mathrm{SaO}_{2}\right)$, end-tidal carbon dioxide (in ventilated patients), and temperature monitoring [24].

Severity of illness, comorbidity and postsurgical status, lack of safety procedural protocol, poor facilities structure, 
TABLE 1: Summary of the characteristics of included studies.

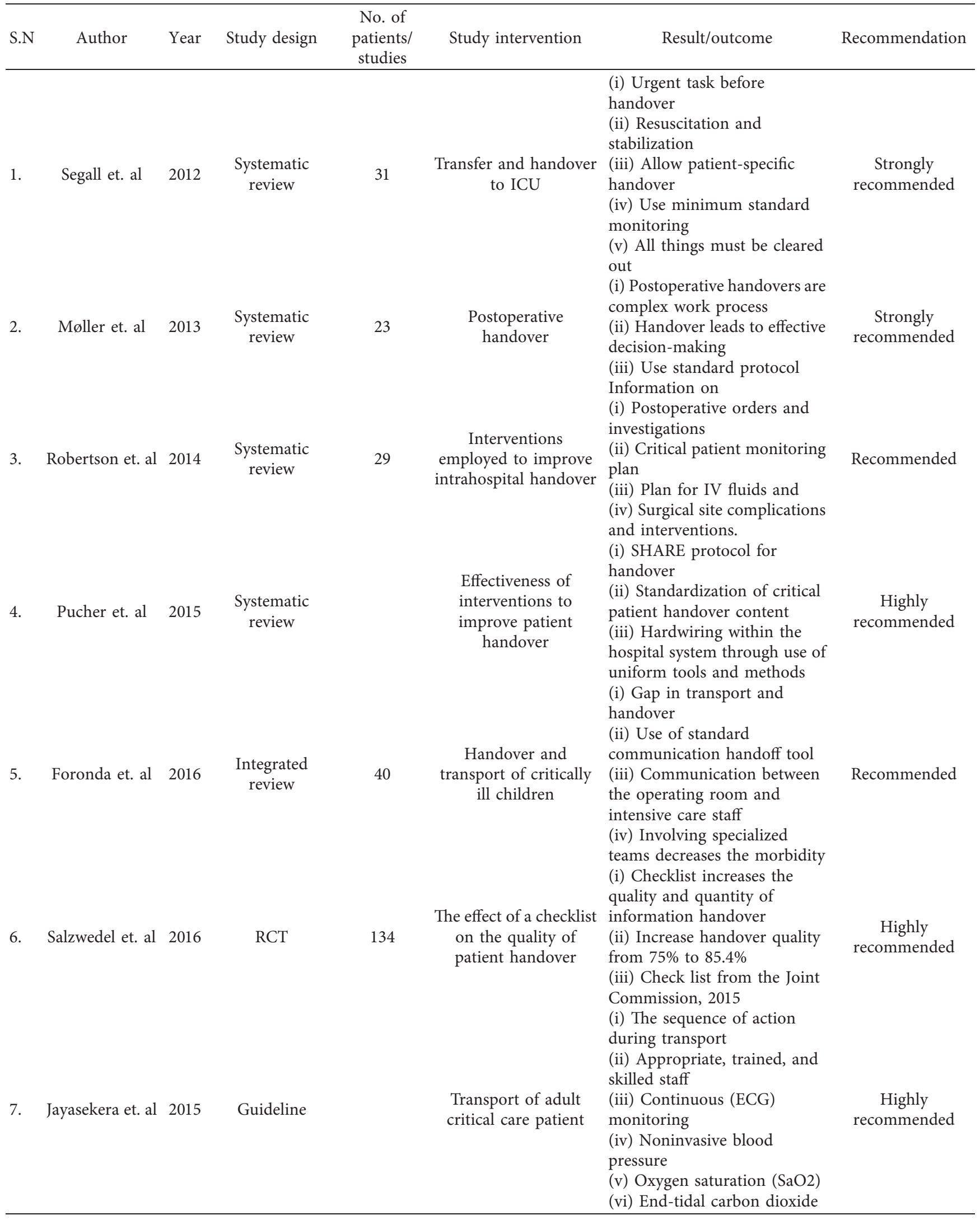


TABle 1: Continued.

\begin{tabular}{|c|c|c|c|c|c|c|c|}
\hline S.N & Author & Year & Study design & $\begin{array}{c}\text { No. of } \\
\text { patients/ } \\
\text { studies }\end{array}$ & Study intervention & Result/outcome & Recommendation \\
\hline 8. & Netes et. al & 2016 & Guideline & & $\begin{array}{c}\text { Critical patient transfer } \\
\text { indication/admission } \\
\text { to ICU }\end{array}$ & $\begin{array}{l}\text { (i) Need of intensive care } \\
\text { therapies } \\
\text { (ii) Need of invasive ventilation } \\
\text { (iii) Need of continuous } \\
\text { invasive hemodynamic } \\
\text { monitoring } \\
\text { (iv) Require life support } \\
\text { therapy for organ failure } \\
\text { (v) Need of intensive } \\
\text { monitoring and therapies only } \\
\text { provided in the ICU } \\
\text { (i) Sources of }\end{array}$ & $\begin{array}{l}\text { Highly } \\
\text { recommended }\end{array}$ \\
\hline 9. & $\begin{array}{l}\text { New Zealand } \\
\text { college of } \\
\text { anesthesia }\end{array}$ & 2015 & Guideline & & $\begin{array}{l}\text { Guidelines for the } \\
\text { transport of critically } \\
\text { ill patients }\end{array}$ & $\begin{array}{l}\text { (i) Sources of oxygen and } \\
\text { airway equipment } \\
\text { (ii) Difficult airway equipment } \\
\text { (iii) Use of standard monitors } \\
\text { (iv) Emergency and patient- } \\
\text { specific medications }\end{array}$ & $\begin{array}{l}\text { Highly } \\
\text { recommended }\end{array}$ \\
\hline 10. & Knight et. al & 2015 & Cohort & 102 & $\begin{array}{c}\text { Factors for } \\
\text { complication during } \\
\text { critical patient } \\
\text { transport }\end{array}$ & $\begin{array}{l}\text { (i) Severity of illness, } \\
\text { comorbidity and postsurgical } \\
\text { status } \\
\text { (ii) Lack of safety procedural } \\
\text { protocol } \\
\text { (iii) Poor facilities structure, } \\
\text { length of transfer } \\
\text { (iv) Availability of monitoring } \\
\text { and equipment } \\
\text { (v) Poor communication, } \\
\text { inadequate training, } \\
\text { insufficient staffing }\end{array}$ & Recommended \\
\hline 11. & Swickard et. al & 2018 & $\begin{array}{l}\text { Retrospective } \\
\text { cohort study }\end{array}$ & 50 & $\begin{array}{c}\text { Patient safety events } \\
\text { during critical care } \\
\text { Transport }\end{array}$ & $\begin{array}{l}\text { (i) Adverse event during } \\
\text { transport }\end{array}$ & Recommended \\
\hline 12. & Nagpal et. al & 2010 & $\begin{array}{l}\text { Prospective } \\
\text { cohort study }\end{array}$ & 65 & $\begin{array}{l}\text { Postoperative } \\
\text { handover }\end{array}$ & $\begin{array}{l}\text { Required information } \\
\text { (i) Name } \\
\text { (ii) Age, weight } \\
\text { (iii) History of allergies } \\
\text { (iv) Diagnosis and procedure } \\
\text { performed } \\
\text { (v) General condition of the } \\
\text { patient } \\
\text { (vi) Previous medical history } \\
\text { (vii) Any coexisting disease }\end{array}$ & Recommended \\
\hline
\end{tabular}

TABLE 2: Levels of evidence and degree of recommendation, Good clinical practice, GCP, WHO, 2011.

\begin{tabular}{lcc}
\hline Level & Type of evidence & Degree of recommendation \\
\hline $1 \mathrm{a}$ & Evidence-based guideline, systematic reviews of RCTs & Strongly recommended/directly applicable \\
$1 \mathrm{~b}$ & Systematic review & Highly recommended/directly applicable \\
$1 \mathrm{c}$ & Randomized clinical trials/RCTs & Recommended/applicable \\
$\mathrm{2a}$ & Systematic reviews of case control or cohort studies. & Extrapolated evidence from other studies \\
$3 \mathrm{a}$ & Nonanalytic studies, e.g., case reports and case series & Extrapolated evidence from other studies \\
\hline
\end{tabular}

Source: Good clinical practice, GCP, WHO, 2011.

length of transfer, availability of monitoring, poor communication, inadequate training, insufficient staffing, and lack of supervision were associated with complication during critical patient transport [25]. Literatures recommended that oxygen, face mask, self-inflating bag, suction equipment, intubation equipment, difficult airway 


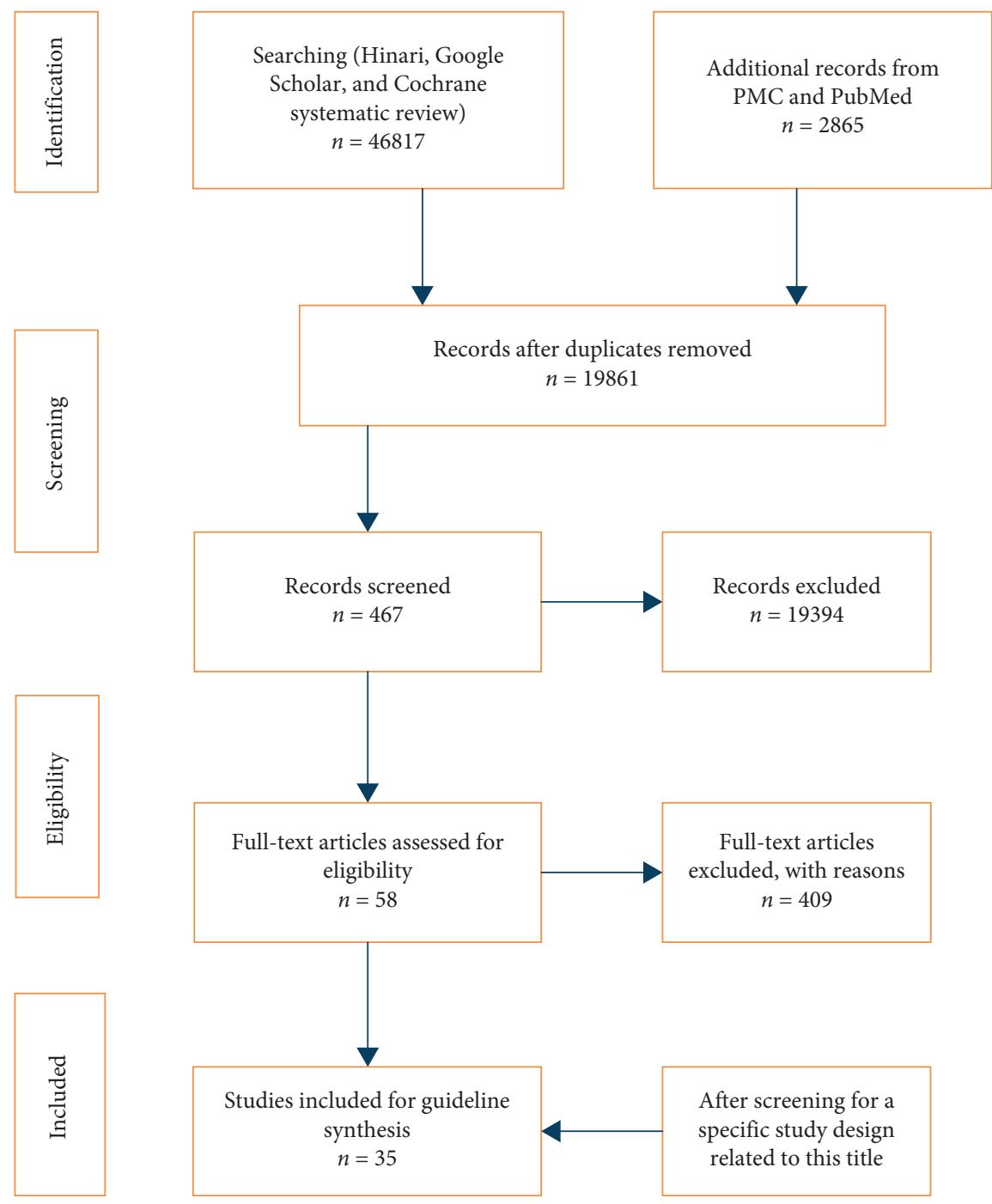

FIGURE 1: PRISMA 2009 Flow Diagram searching.

\section{Evidence-based guideline on patient transport and handover to ICU}

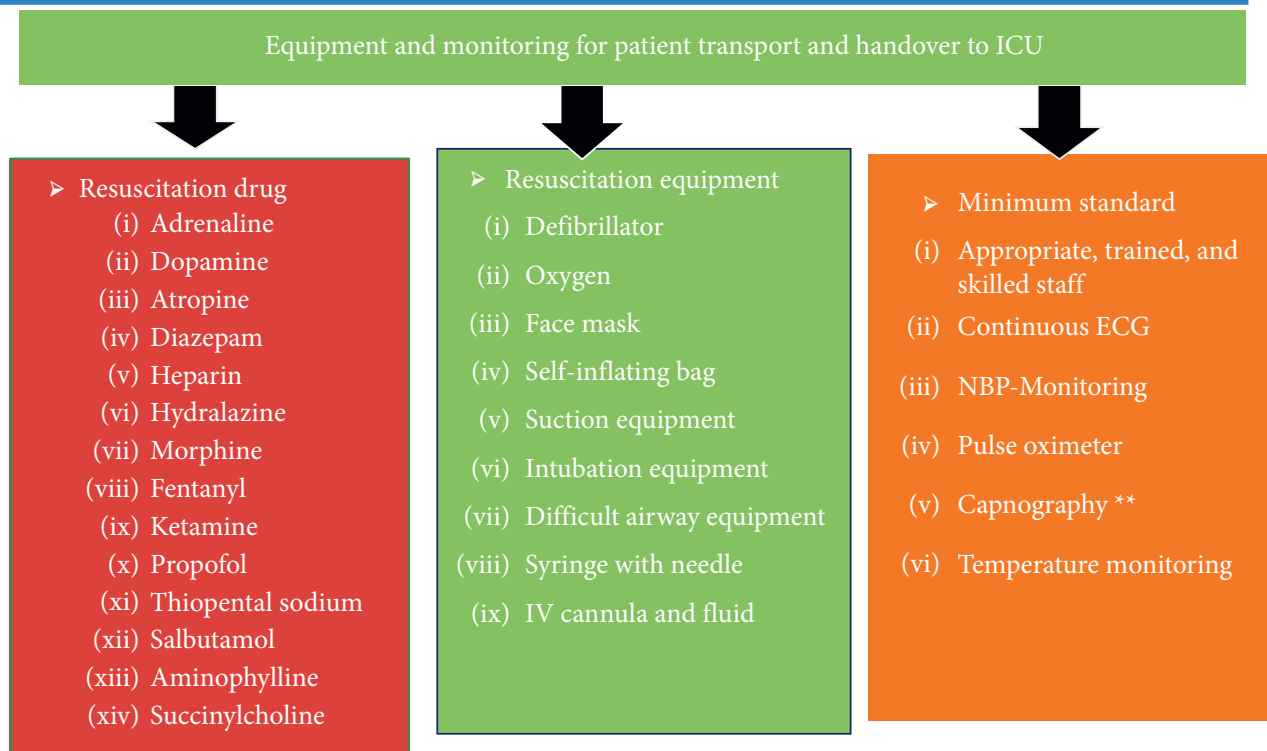

NB. ${ }^{* *}$ indicates optional based on availability

Figure 2: Equipment and monitoring needed for patient transport and hand over to ICU. 


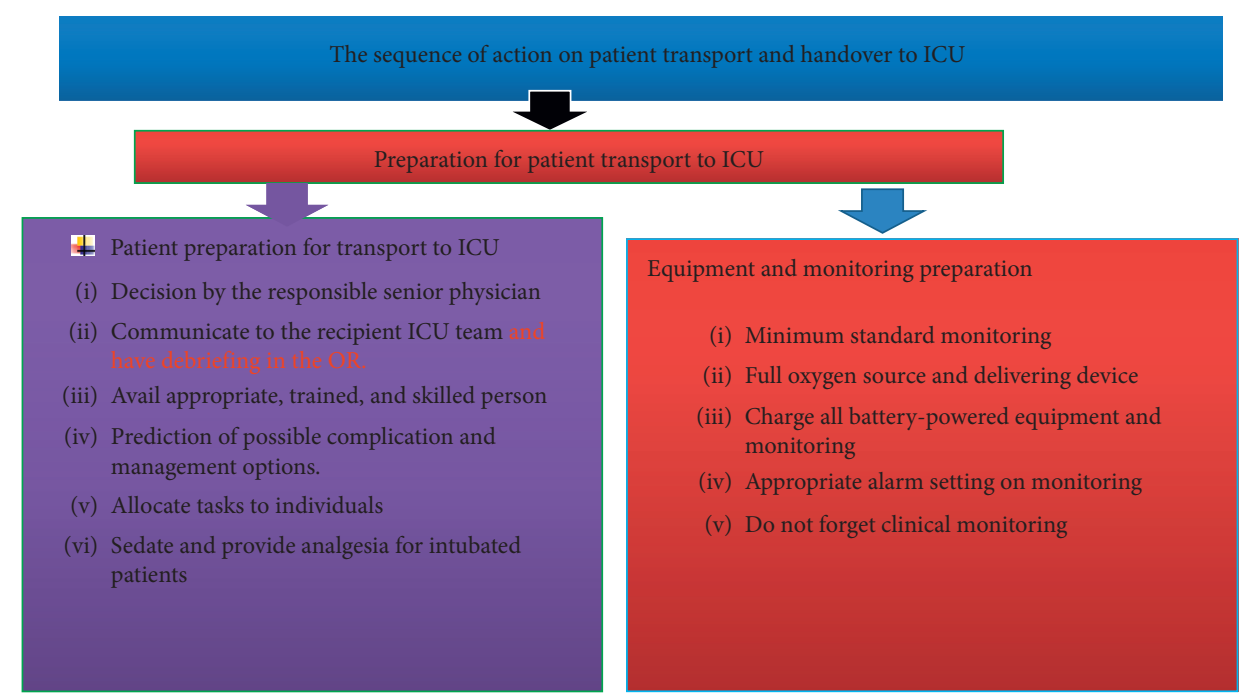

FIgURE 3: Sequence of activities during critical patient transfer and and over to ICU.
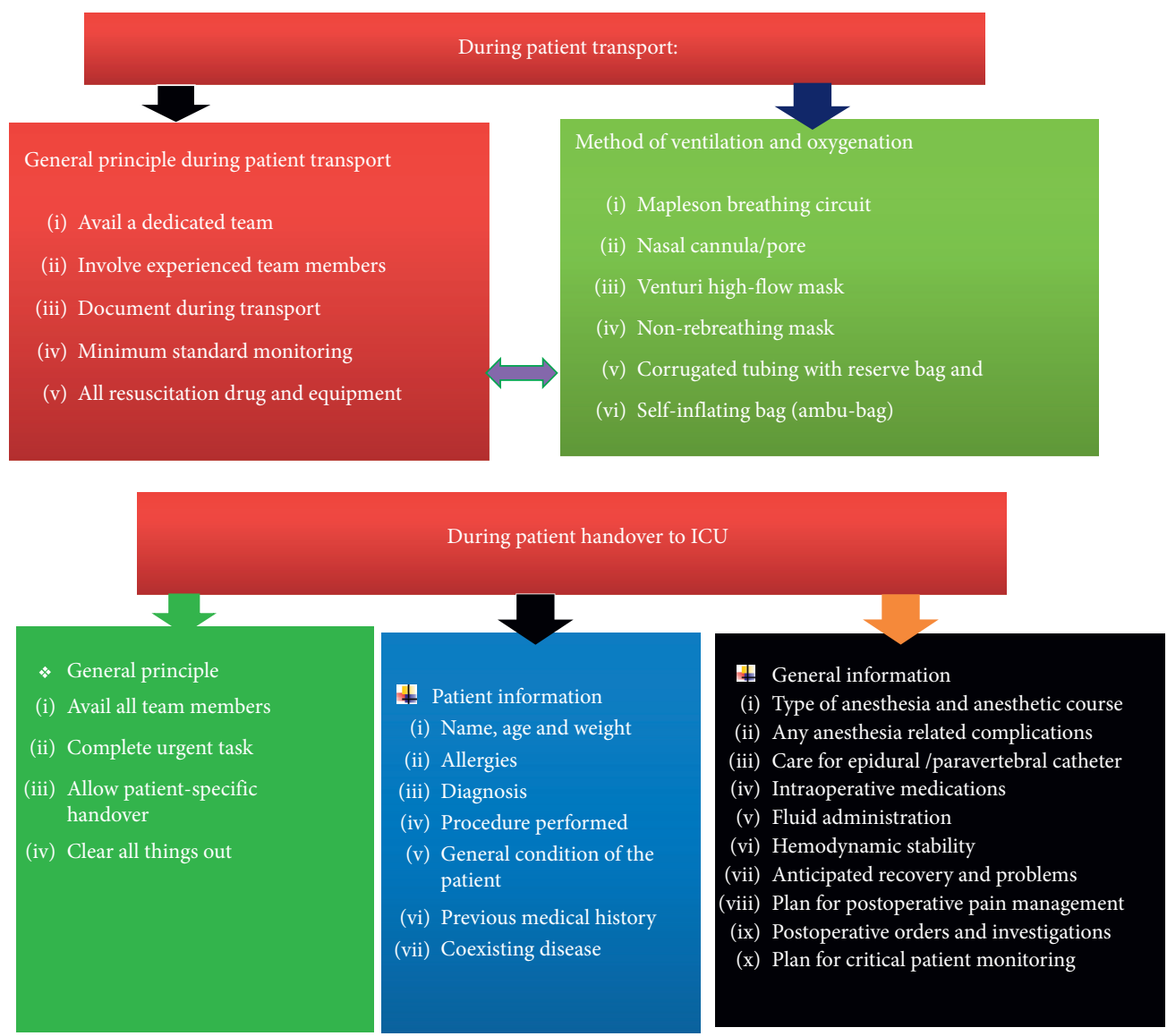

FIGURE 4: Patient monitoring and mode of ventilation on patient transfer to ICU.

equipment, syringe with needle, NIBP monitoring, pulse oxymetery, capnography, ECG, and all drugs necessary to manage acute life-threatening medical emergencies should be readily available during transport $[23,26]$.

One randomized control trial found that the amount of important information transmitted during handover increased from $75 \%$ in the control group compared with $87.1 \%$ in the study group when they used a standardized checklist [27]. A cohort study conducted in Germany showed that postoperative residual neuromuscular blockade was strongly associated with ICU admission [28]. Handover includes patient information such as name, age, weight, 


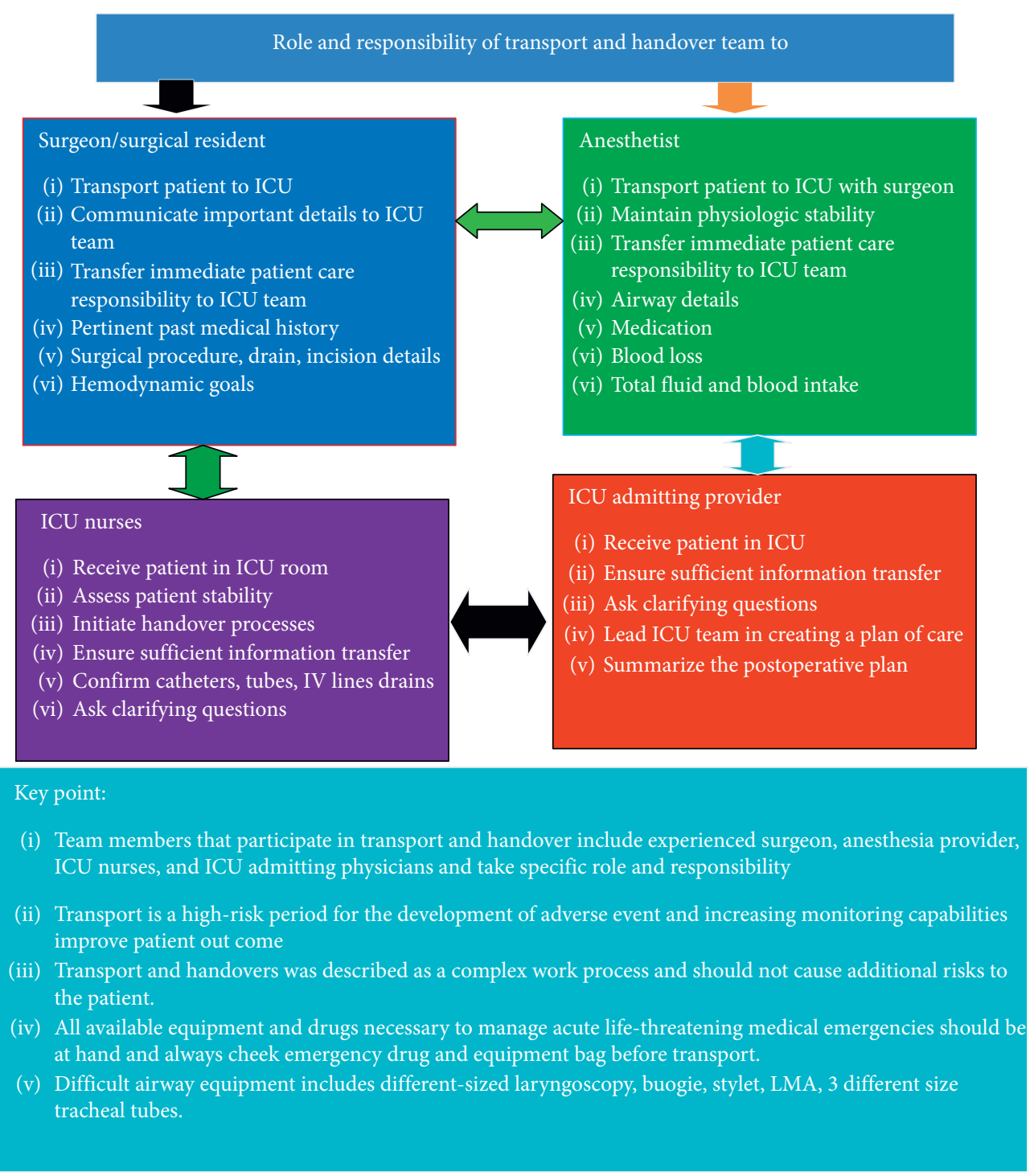

Figure 5: Roles and responsibilities of the critical care givers during patient handover to ICU.

allergies, diagnosis, procedure performed, general condition of the patient, previous medical history, and any coexisting disease [15]. Type of anesthesia, any intraoperative events, medications, IV fluids, estimated blood loss, critical patient monitoring plan, and plan for IV fluids should be handed over appropriately [29]. Surgical site dressings, tubes, drains, and packing; surgical complications; and interventions should be included in the handover phase [30].

The German Association of Anesthesiology and Intensive Care recommends the genuine relationship between organizational prerequisites, professional skills, behaviors, and actions to perform safe transfer patients to ICU [31]. The team consists of surgeon, anesthetist, ICU nurses, and ICU admitting provider participated in transport and handover [29]. Intensive care physician summarizes the postoperative plan in the presence of the ICU team, surgeon, and anesthesiologist [32].

\section{Conclusion}

The transfer and handover of the critically ill patient are not standardized throughout even though some inconsistent guidelines are available. Maintaining adequate sedation and analgesia throughout transport can make the patient calm and comfortable and help avoid unnecessary interruption during transport; however, patient instability and inadequate training of the care provider may increase the risk for interruption [33]. In contrast, a randomized controlled study shows an increased ICU stay and hypoxia in mechanical ventilated patient in sedated patients [34]. 
Guidelines showed that the minimum standards of monitoring required during critical patient transport include appropriate trained and skilled staff, continuous cardiac rhythm (ECG) monitoring, noninvasive blood pressure, oxygen saturation $\left(\mathrm{SaO}_{2}\right)$, end-tidal carbon dioxide (in ventilated patients), and temperature monitoring [24]. Another study does not recommend routine monitoring of end-tidal CO2 during short transport times in adult patients [35].

Manual ventilation delivers unpredictable tidal volumes [36] and transport ventilators consistently deliver the expected tidal volume, but their battery duration has been questioned [37]. A randomized trial argued the high increase in end-tidal $\mathrm{CO}_{2}$ with bag-mask ventilation [38] against another study that showed no significant change from the baseline [39].

A decision to transfer critical patients should be made by senior physicians in the unit after full assessment and communication between referring and the receiving team. Transporting patients is a risky procedure, and it requires good communication, planning, and appropriate staffing. Any patient who requires transportation must be effectively stabilized before departure. Planning and preparation for critical patient transport to ICU are vital to prevent adverse events.

For better patient outcome and safety, use of minimum standard monitoring devices is advisable. Critical patient transport to ICU should be performed early for indicated patients in the postoperative period. Adequate prevention of residual neuromuscular block may decrease rates of unplanned intensive care unit admission. All team members who participate in transport and handover take specific role and responsibility Figures 2-5.

\section{Conflicts of Interest}

The authors declare no conflicts of interest.

\section{References}

[1] N. Siddiqui, C. Arzola, M. Iqbal et al., "Deficits in information transfer between anaesthesiologist and postanaesthesia care unit staff," European Journal of Anaesthesiology, vol. 29, no. 9, pp. 438-445, 2012.

[2] C. Schwebel, C. Clec'h, S. Magne et al., "Safety of intrahospital transport in ventilated critically ill patients," Critical Care Medicine, vol. 41, no. 8, pp. 1919-1928, 2013.

[3] F. B. John, C. M. David, and D. W. John, Morgan \& Mikhails Clinical Anesthesiology, Mcgraw-Hill, New York, NY, USA, 2018.

[4] J. J. Ligtenberg, L. G. Arnold, Y. Stienstra et al., "Quality of interhospital transport of critically ill patients: a prospective audit," Critical Care, vol. 9, no. 4, p. R446, 2005.

[5] D. Lahner, A. Nikolic, P. Marhofer et al., "Incidence of complications in intrahospital transport of critically ill patients--experience in an Austrian university hospital," Wiener Klinische Wochenschrift, vol. 119, no. 13-14, pp. 412-416, 2007.

[6] V. C. Veiga, N. F. Postalli, T. K. Alvarisa et al., "Adverse events during intrahospital transport of critically ill patients in a large hospital," Revista Brasileira de terapia intensiva, vol. 31, no. 1, pp. 15-20, 2019.

[7] W. Habre and F. Peták, "Perioperative use of oxygen: variabilities across age," British Journal of Anaesthesia, vol. 113, no. Suppl 2, pp. ii26-ii36, 2014.

[8] N. Segall, A. S. Bonifacio, R. A. Schroeder et al., "Can we make postoperative patient handovers safer? A systematic review of the literature," Anesthesia \& Analgesia, vol. 115, no. 1, pp. 102-115, 2012.

[9] S. A. Jeffcott, S. M. Evans, P. A. Cameron, G. S. M. Chin, and J. E. Ibrahim, "Improving measurement in clinical handover," Quality and Safety in Health Care, vol. 18, no. 4, pp. 272-276, 2009.

[10] A. L. P. Vieira, A. M. N. dos Santos, M. K. Okuyama, M. H. Miyoshi, M. F. B. d. Almeida, and R. Guinsburg, "Factors associated with clinical complications during intrahospital transports in a neonatal unit in Brazil," Journal of Tropical Pediatrics, vol. 57, no. 5, pp. 368-374, 2010.

[11] S. Kumar, A. R. McKean, A. Ramwell, C. Johnston, and S. Leaver, "Optimizing postoperative handover to the intensive care unit at a tertiary centre," British Journal of Hospital Medicine, vol. 78, no. 1, pp. 12-15, 2005.

[12] S. Bourn, S. Wijesingha, and G. Nordmann, "Transfer of the critically ill adult patient," BJA Education, vol. 18, no. 3, pp. 63-68, 2018.

[13] M. L. Mitchell and M. Courtney, "Improving transfer from the intensive care unit: the development, implementation and evaluation of a brochure based on Knowles' Adult Learning Theory," International Journal of Nursing Practice, vol. 11, no. 6, pp. 257-268, 2005.

[14] Whiteley, Guidelines for the Transport of the Critically Ill Adult, Whiteley, Auckland, New Zealand, third edition, 2019.

[15] K. Nagpal, S. Arora, M. Abboudi et al., "Postoperative handover," Annals of Surgery, vol. 252, no. 1, pp. 171-176, 2010.

[16] A. S. Bonifacio, N. Segall, A. Barbeito, J. Taekman, R. Schroeder, and J. B. Mark, "Handovers from the or to the ICU," International Anesthesiology Clinics, vol. 51, no. 1, pp. 43-61, 2013.

[17] L. M. McElroy, K. R. Macapagal, K. M. Collins et al., "Clinician perceptions of operating room to intensive care unit handoffs and implications for patient safety: a qualitative study," The American Journal of Surgery, vol. 210, no. 4, pp. 629-635, 2015.

[18] T. P. Møller, M. D. Madsen, L. Fuhrmann, and D. Østergaard, "Postoperative handover: characteristics and considerations on improvementA systematic review," European Journal of Anaesthesiology (EJA), vol. 30, no. 5, pp. 229-242, 2013.

[19] K. R. Catchpole, M. R. De Leval, A. McEwan et al., "Patient handover from surgery to intensive care: using Formula 1 pitstop and aviation models to improve safety and quality," Pediatric Anesthesia, vol. 17, no. 5, pp. 470-478, 2007.

[20] P. H. Pucher, M. J. Johnston, R. Aggarwal, S. Arora, and A. Darzi, "Effectiveness of interventions to improve patient handover in surgery: a systematic review," Surgery, vol. 158, no. 1, pp. 85-95, 2015.

[21] R. Melamed, L. L. Boland, J. P. Normington et al., "Postoperative respiratory failure necessitating transfer to the intensive care unit in orthopedic surgery patients: risk factors, costs, and outcomes," Perioperative Medicine (London, England), vol. 5, p. 19, 2016.

[22] J. L. Nates, M. Nunnally, R. Kleinpell et al., "ICU admission, discharge, and triage guidelines," Critical Care Medicine, vol. 44, no. 8, pp. 1553-1602, 2016. 
[23] S. Gupta, A. Bhagotra, S. Gulati, and J. Sharma, "Guidelines for the transport of critically Ill patients," JK Science, vol. 6, no. 2, pp. 109-112, 2004.

[24] D. C. Jayasekera, D. B. Goonathillake, D. S. Hapuarachchi et al., Guidelines for Transport of Adult Critical Care Patient in Sri Lanka November, Critical Care of Medicine, Sri Lanka, 2015.

[25] P. H. Knight, N. Maheshwari, J. Hussain et al., "Complications during intrahospital transport of critically ill patients: focus on risk identification and prevention," International Journal of Critical Illness and Injury Science, vol. 5, no. 4, pp. 256-264, 2015.

[26] Anaesthesia aanzco. guidelines for transport of critically ill patients 2015.

[27] C. Salzwedel, V. Mai, M. A. Punke, S. Kluge, and D. A. Reuter, "The effect of a checklist on the quality of patient handover from the operating room to the intensive care unit: a randomized controlled trial," Journal of Critical Care, vol. 32, pp. 170-174, 2016.

[28] S. D. Grabitz, N. Rajaratnam, K. Chhagani et al., "The effects of postoperative residual neuromuscular blockade on hospital costs and intensive care unit admission," Anesthesia \& Analgesia, vol. 128, no. 6, pp. 1129-1136, 2019.

[29] M. B. Lane-Fall, J. L. Pascual, S. Massa et al., "Developing a standard handoff process for operating room-to-ICU transitions: multidisciplinary clinician perspectives from the handoffs and transitions in critical care (HATRICC) study," The Joint Commission Journal on Quality and Patient Safety, vol. 44, no. 9, pp. 514-525, 2018.

[30] E. Funk, B. Taicher, J. Thompson, K. Iannello, B. Morgan, and S. Hawks, "Structured handover in the pediatric postanesthesia care unit," Journal of PeriAnesthesia Nursing, vol. 31, no. 1, pp. 63-72, 2016.

[31] V. Dossow and B. Zwissler, "Recommendations of the German Association of Anesthesiology and Intensive Care Medicine (DGAI) on structured patient handover in the perioperative setting: the SBAR concept," Der Anaesthesist, vol. 65, no. Suppl 1, pp. 1-4, 2016.

[32] D. S. Wheeler, A. M. Sheets, and F. C. Ryckman, "Improving transitions of care between the operating room and intensive care unit," Translational Pediatrics, vol. 7, no. 4, p. 299, 2018.

[33] D. Johnston, K. Franklin, P. Rigby, K. Bergman, and S. B. Davidson, "Sedation and analgesia in transportation of acutely and critically ill patients," Critical Care Nursing Clinics of North America, vol. 28, no. 2, pp. 137-154, 2016.

[34] E. Laerkner, T. Stroem, and P. Toft, "No-sedation during mechanical ventilation: impact on patient's consciousness, nursing workload and costs," Nursing in Critical Care, vol. 21, no. 1, pp. 28-35, 2016.

[35] S. C. Palmon, M. Liu, L. E. Moore, and J. R. Kirsch, "Capnography facilitates tight control of ventilation during transport," Critical Care Medicine, vol. 24, no. 4, pp. 608-611, 1996.

[36] H. W. E. B. GervaisD. Konietzke et al., "Comparison of blood gases of ventilated patients during transport," Crit Care Med, vol. 15, pp. 761-763, 2000.

[37] S. G. M. Boussen and P. Michelet, "Evaluation of Ventilators Used during Transport of Critically Ill Patients: A Bench Study," Respiratory Care, vol. 58, no. 11, pp. 1911-1922, 2013.

[38] E. O. O’Brien, B. J. Newhouse, B. Cronin et al., "Hemodynamic consequence of hand ventilation versus machine ventilation during transport after cardiac surgery," Journal of Cardiothoracic and Vascular Anesthesia, vol. 31, no. 4, pp. 1246-1249, 2017.
[39] M. H. Stroud, P. Prodhan, M. Moss, R. Fiser, S. Schexnayder, and K. Anand, "Enhanced monitoring improves pediatric transport outcomes: a randomized controlled trial," Pediatrics, vol. 127, no. 1, pp. 42-48, 2011. 